JULIA SILVA BENETI

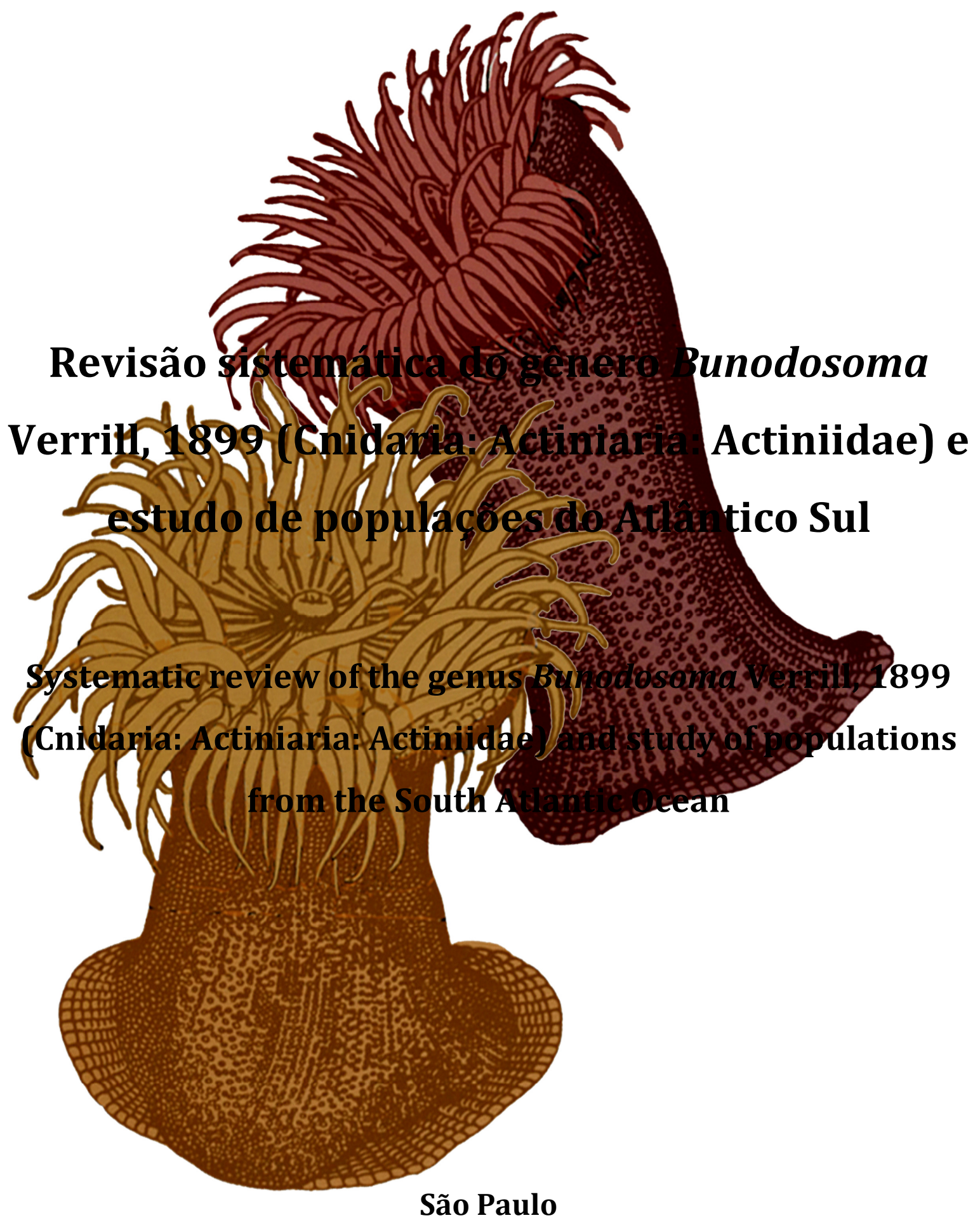


Julia Silva Beneti

\section{Revisão sistemática do gênero Bunodosoma Verrill, 1899 (Cnidaria: Actiniaria: Actiniidae) e estudo de populações do Atlântico Sul}

\footnotetext{
Systematic review of the genus Bunodosoma Verrill, 1899(Cnidaria: Actiniaria: Actiniidae) and study of populations from the South Atlantic Ocean
}

Tese apresentada ao Instituto de Biociências da Universidade de São Paulo, para a obtenção de Título de Doutor em Ciências Biológicas na Área de Zoologia.

Orientador: Prof. Dr. André Carrara Morandini

Coorientadora: Dra. Luciana C. F. R. de Gusmão

São Paulo 
Beneti, Julia Silva

Revisão sistemática do gênero Bunodosoma Verrill, 1899 (Cnidaria: Actiniaria: Actiniidae) e estudo de populações do Atlântico Sul

$236 \mathrm{p}$

Tese (Doutorado) - Instituto de Biociências da Universidade de São Paulo. Departamento de Zoologia.

1. Anêmonas do mar 2. Delimitação de espécies 3. Delimitação de populações

I. Universidade de São Paulo. Instituto de Biociências. Departamento de Zoologia.

Comissão Julgadora:

Prof(a). Dr (a).

Prof(a). Dr (a).

Prof(a). Dr (a).

$\operatorname{Prof(a).~Dr~(a).~}$

Prof. Dr. André Carrara Morandini

Orientador 


\section{Agradecimentos}

Ao meu orientador, Prof. Dr. André Carrara Morandini, por sua enorme contribuição na minha formação científica e por aceitar a me orientar mesmo eu não querendo estudar medusas! Além disso, agradeço pela grande ajuda nas coletas, pelas conversas e por corrigir meus textos e responder e-mails super rápido!

Aos colegas e amigos, presentes e passados, do Laboratório de Cultivo e Estudos em Cnidaria, Renato Nagata, Gisele Tiseo, Henrique Alves, Leandro Santos, Clarissa Molinari, Edgar Gamero, Mayara Jordano e Jonathan Lawley, pela divertida convivência (comigo e com a minha bagunça). E em especial ao Sérgio N. Stampar e Max M. Maronna pela paciência para me ensinar e ajudar infinitamente com os estudos moleculares.

À minha co-orientadora Luciana Gusmão, pela ajuda em coletas e no laboratório, por me acolher em Recife e em Nova Iorque, e pela amizade (e a ioga, é claro).

À Dra. Estefania Rodríguez, que me recebeu e disponibilizou toda a estrutura em seu Laboratório no American Museum of Natural History, e me ajudou a compreender várias coisas que eu não entendia ainda sobre as lindas anêmonas.

À todos os funcionários do Instituto de Biociências, em especial ao Ênio, Manuel, Bia, Sabrina e Phillipe por me salvarem inúmeras vezes ao longo desses 4 anos e meio, e me ajudarem prontamente com dúvidas de histologia ou molecular que foram surgindo pelo caminho! E à Lilian e Erika pela enorme ajuda nas questões burocráticas!

A todo mundo que me ajudou em coletas: André Morandini, Sérgio Stampar, Karla Paresque, Luciana Gusmão, Renato Nagata, Nathalia Padovanni, Thomás Banha, Alexandre Nascimento, Felipe Rocha, Nathalia Hristov, María Mendoza Becerril, Amanda Cunha, Fabián Acuña, Agustín Garese, Fabrizio Scarabino, Valentina Leoni, Romina Trinchin e Bárbara Romera, e muitas outras pessoas.

Ao Prof. Dr. Fabián Acuña e Dr. Agustín Garese pela ajuda com o estudo dos cnidocistos e pela ótima recepção em Mar del Plata. E à Irene Deserti por tornar essa semana na Argentina muito agradável com os passeios. 
À Profa. Dra. Vera Solferini e seus alunos, principalmente Fernanda, Cecília e Elen, por me ajudarem com as análises de população.

Aos amigos da Zoo e agregados, pelas conversas, boicotes ao Bobó, Churras da Zoo, CVZoos, e tudo mais que aconteceu nesses 4 anos e meio, além da ajuda na tese: Pedro H., Jorge, Loboda, Bárbara, Pedro G., Isa, Rach, Mari, Rafa, Adri, Alípio, Jhon, Anne, Juan, Thalles, Avatar, Gabriel (os dois), Carol (as duas), Victor, Gordo, Bruna, Bala, Tama, Nilvea, Caios peixes e Antunes, etc.

A todas minhas companheiras de casa, que dividiram comigo meus e seus momentos de alegria, fofocas e estresse: Ruli, Paula, Alejandra, Nati, Flávia, Melina, Camila, Natália, Vivi.

Às minhas amigas queridas (entre outras coisas), que mesmo longe estão sempre perto ciberneticamente, falando besteira e contando histórias de mundos longínquos: Rach, Ruli, Cobrinha, Esther e Paula.

Aos melhores amigos da M11, simplesmente por existirem e por ser a melhor turma que alguém pode ter! À Sofia, melhor amiga pra dividir coisas do doutorado e da vida! E à Tati, que de uma forma ou de outra está desde sempre presente em tudo que eu faço na vida, seja com palavras inteligentes ou de carinho pra dar!

À Nara, a gata mais charmosa e cheia de personalidade que já existiu, por me fazer companhia todo santo dia (dormindo, mas tudo bem!), principalmente agora no fim da tese.

À minha família maravilhosa e divertida, Cesar, Claudia, Lucas e Sara, sempre presentes e bem humorados, dando forças nos momentos em que mais preciso. Nada de nada na minha vida seria possível sem vocês. E um agradecimento mais do que especial e merecido pra pequena irmã Sara que editou grande parte das fotos dessa tese (quase) sem reclamar. E à minha família canina Chico, Bela, Malu, Frida, por me fazerem companhia na sala de casa enquanto eu editava minhas tabelas infinitas de cnidocistos (Zeca, Lia, Nina, Meg e Mila estavam lá zoando em espírito, certamente).

Obrigada a todos!!! 


\section{Índice}

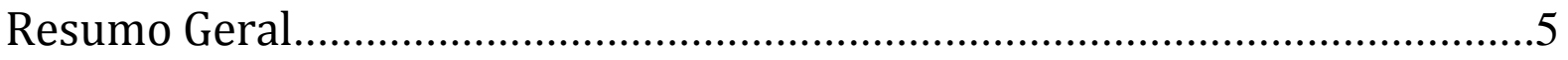

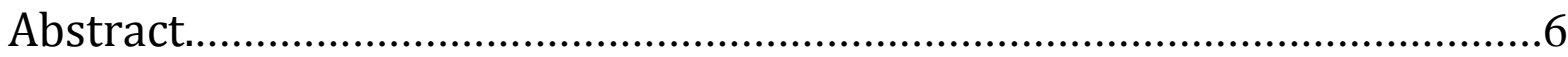

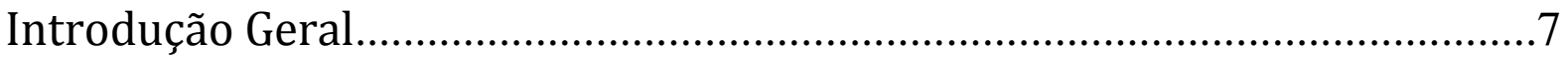

Capítulo 1

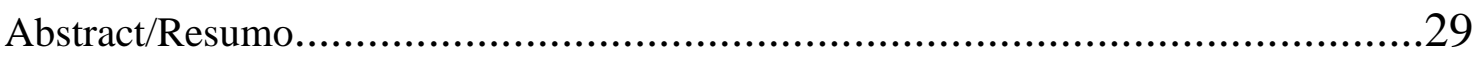

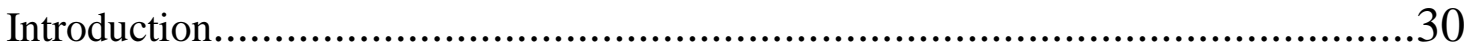

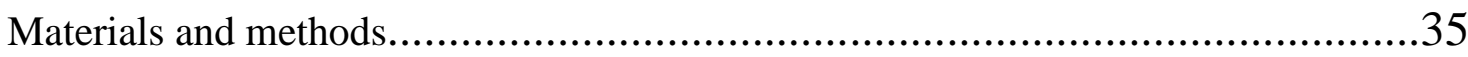

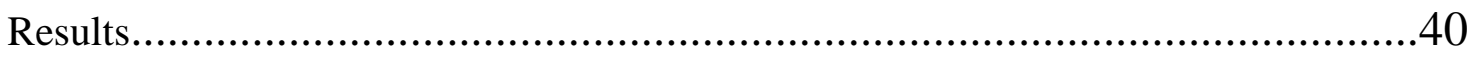

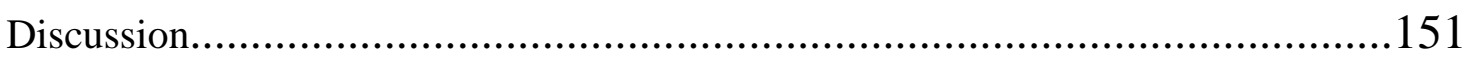

Capítulo 2.

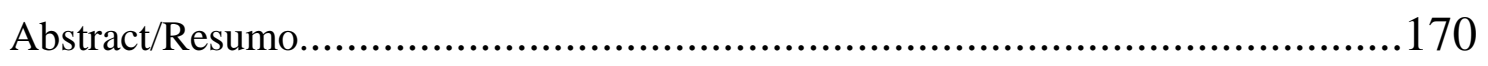

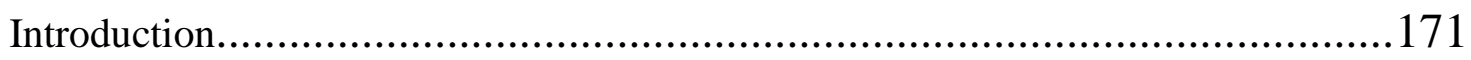

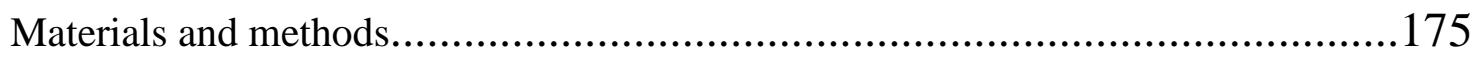

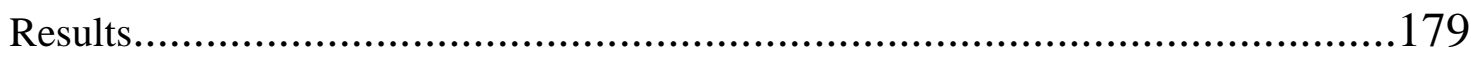

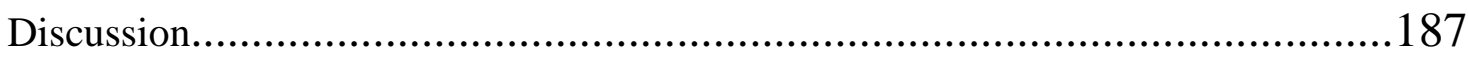

Capítulo 3

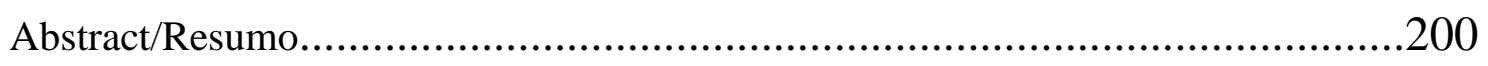

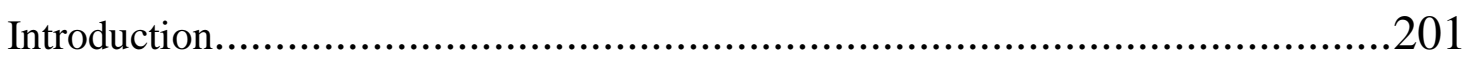

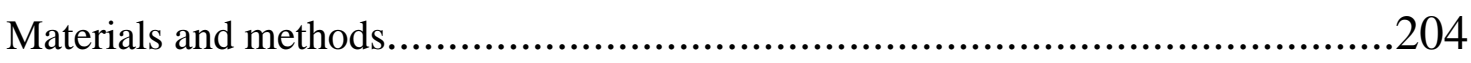

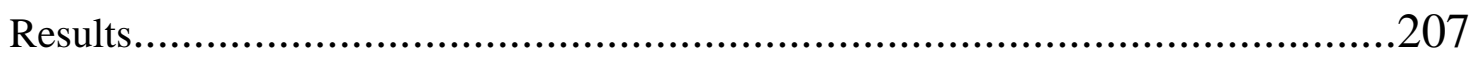

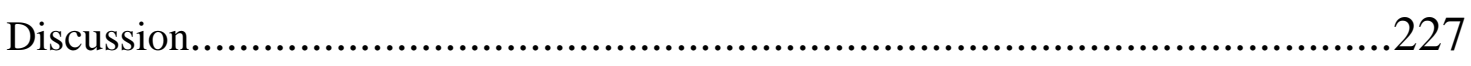

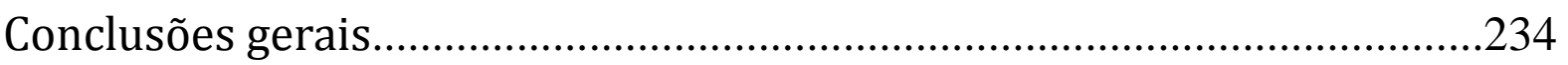




\section{Resumo}

Bunodosoma é um gênero cosmopolita de anêmonas que apresentam pólipos grandes e conspícuos, sendo comuns em ambientes costeiros. Atualmente compreende 14 espécies válidas e, apesar de estas serem utilizadas em estudos filogenéticos dentro da ordem Actiniaria, não existem abordagens visando resolver os problemas intragenéricos, e o conhecimento da diversidade do gênero é limitado por problemas taxonômicos e de identificação. Dessa forma, é necessário que se estabeleçam os limites entre a variação inter e intraespecífica dos pólipos, e para isso devem-se integrar informações morfológicas e moleculares. As espécies B. cangicum, B. caissarum e B. zamponii, endêmicas do Atlântico Sul e com diversos registros ao longo da costa, são um interessante modelo para o estudo destas variações. Portanto, os objetivos desse projeto são: 1) realizar a revisão sistemática e propor uma hipótese filogenética para o gênero Bunodosoma com base em evidências moleculares; 2) explorar a viabilidade da utilização do marcador molecular ITS nos estudos de populações de anêmonas e testar outros marcadores nucleares que possam representar as diferenças genéticas inter e intraespecíficas; e 3) verificar a variabilidade morfológica (utilizando dados de tamanhos de cnidocistos) e molecular intraespecífica das espécies $B$. caissarum e B. cangicum. A revisão sistemática, apresentada no Capítulo 1, evidenciou que 14 espécies podem ser reconhecidas, apesar de o status taxonômico de três delas permanecer aberto a testes. A análise filogenética com os dados moleculares disponíveis não fornece evidência para o monofiletismo de Bunodosoma, que foi recuperado parafilético em relação a outras espécies da família Actiniidae. No Capítulo 2, as análises filogenéticas e filogeográficas com o marcador ITS forneceram suporte para o reconhecimento de dois clados: o primeiro composto por indivíduos de B. caissarum e o segundo por indivíduos de $B$. cangicum e B. zamponii. Apesar de se mostrar útil na delimitação de grandes grupos, o ITS é um marcador eficiente na delimitação de populações. Em relação à variação morfométrica dos cnidocistos, os resultados apresentados no Capítulo 3 demonstram que, caso bem empregados e analisados, alguns tipos de nematocistos podem ter valor taxonômico na delimitação de populações de anêmonas. O conjunto dos resultados do presente estudo contribuem para um melhor conhecimento da sistemática e biologia desses organismos, além de gerar subsídios para a compreensão de aspectos evolutivos da ordem Actiniaria.

Palavras-chave: taxonomia, sistemática molecular, populações, cnidocysts. 


\begin{abstract}
Bunodosoma is a cosmopolite genus of sea anemones of large and conspicuous polyps, common in costal environments. It currently comprises 14 species and, despite being employed in phylogenetic studies within the order Actiniaria, no study so far has tried to solve intrageneric problems in Bunodosoma, so the knowledge about the genus' diversity is limited by problems of taxonomy and identification. Thus, it is necessary to establish the limits of inter and intraspecific variation of the polyps. To accomplish that, integrative studies using morphological and molecular data must be carried out. The species B. cangicum, $B$. caissarum, and B. zamponii are endemic of the South Atlantic Ocean. They represent an interesting model to study the aforementioned variation. Therefore, the goals of this study are: 1) to review the systematics and to propose a phylogenetic hypothesis for the genus Bunodosoma based on molecular evidence; 2) to explore the usage of the molecular marker ITS in population studies of sea anemones, as well as to test other nuclear regions that may show inter and intraspecific variation; and 3) to access the phenotypic (based on cnidocyst size variation) and molecular intraspecific variability of $\quad$ B. caissarum and B. cangicum. The systematic review, presented in Chapter 1 , showed that there are 14 recognized species; three of them need further analyses. The molecular phylogenetic analysis did not provide evidences for the monophyly of Bunodosoma, which was recovered paraphyletic regarding other species of the family Actiniidae. In Chapter 2, the phylogenetic and phylogeographic analysis of ITS provided support to recognize two major clades: one composed by $B$. caissarum individuals and the other by individuals of B. cangicum plus B. zamponii. Despite been useful to delimit major groups, the ITS marker is not efficient to delimit populations. Regarding the morphometric variation of the cnidocysts, the results presented in Chapter 3 demonstrated that, when employed with caution, some types may present taxonomic value in population delimitation. The results of the present study contribute to a better understanding of the systematics and the biology of Bunodosoma, and provide support to the comprehension of evolutionary aspects of the order Actiniaria.
\end{abstract}

Key words: taxonomy, molecular systematics, populations, cnidocysts. 


\section{INTRODUÇÃo}

As anêmonas-do-mar (Filo Cnidaria, Classe Anthozoa, Ordem Actiniaria) estão entre os invertebrados mais conspícuos dos ambientes marinhos costeiros, principalmente em costões rochosos e praias arenosas. Tais animais apresentam uma grande diversidade morfológica e ecológica, sendo encontrados em diferentes tipos de habitats marinhos, em todas as profundidades e latitudes, o que faz com que estejam entre os membros mais diversos e bem sucedidos entre os antozoários (Daly et al., 2008).

Dentre os cnidários, os antozoários compreendem aproximadamente 7.500 espécies (Daly et al., 2007), sendo a classe mais diversa do filo. Os antozoários são diagnosticados por apresentarem exclusivamente formas polipoides no ciclo de vida, actinofaringe, sifonóglife e mesentérios (Daly et al., 2007). Atualmente a classe é definida como apresentando duas linhagens monofiléticas caracterizadas basicamente por meio da simetria corporal octâmera, no caso dos octocorais, e hexâmera para os hexacorais (embora variações possam existir). Stampar et al (2014) propõem que a ordem Ceriantharia, tradicionalmente incluída em Hexacorallia, seja considerada como um clado à parte, mas que sua posição dentro de Anthozoa ou de Cnidaria deve ser melhor estudada antes de ser definida.

\section{Ordem Actiniaria}

Um pólipo de anêmona do mar consiste basicamente em uma coluna, estrutura tubular que na maioria das espécies tem grande capacidade de contração, em cuja base (porção proximal) geralmente há uma estrutura de adesão ao substrato, o disco pedal. A porção distal 
da coluna é o disco oral, na qual há uma boca oval ou em forma de fenda circundada pelo perístoma. Na ordem Actiniaria, a coluna pode apresentar estruturas (verrugas, vesículas, esférulas marginais, etc.), divisões (escapo, escapulo, capítulo, etc.) ou pode ser lisa. Na margem distal da coluna estão os tentáculos, que estão frequentemente dispostos em ciclos em torno do perístoma.

Internamente à boca em sentido distal-proximal segue a actinofaringe, uma estrutura tubular e lateralmente comprimida que faz a comunicação do ambiente externo com a cavidade gastrovascular. Esta apresenta fundo cego e é multifuncional, pois atua na digestão extracelular, circulação, excreção, reprodução e suporte por esqueleto hidrostático (Shick, 1991). As anêmonas apresentam simetria corporal bilateral ou birradial, evidenciada pelo fato de terem a faringe comprimida e pela presença de um ou dois sifonóglifes, sulcos ciliados que têm início em cantos opostos da boca e que se estendem ao longo do comprimento da faringe.

A cavidade gastrovascular é dividida em câmaras radiais por mesentérios, que são dobras longitudinais de gastroderme (endoderme) e mesogléia. Os prolongamentos distais das câmaras radiais formam os tentáculos ocos. Os tentáculos dos ciclos mais internos são denominados endocélicos, pois suas cavidades são contínuas com as câmaras formadas por mesentérios do mesmo par, denominadas endoceles. Já os tentáculos do último ciclo são exocélicos, por estarem ligados às exoceles, espaços entre mesentérios de pares distintos.

Os mesentérios ocorrem aos pares e podem ser de dois tipos: completos ou perfeitos, são os que se ligam à parede da coluna da anêmona e à actinofaringe; ou incompletos ou imperfeitos, aqueles que se ligam apenas à parede da coluna da anêmona. A margem do mesentério é denominada filamento mesentérico, e é formada por três regiões: de ambos os lados os tratos ciliados e no meio o trato cnidoglandular. Atrás do filamento mesentérico está a porção do mesentério na qual o tecido reprodutivo se desenvolve. O número de mesentérios 
completos ou incompletos e a presença de tecido reprodutivo em determinados mesentérios constitui um caráter taxonômico relevante.

Os principais músculos utilizados na taxonomia de anêmonas do mar são o esfíncter marginal e os músculos retratores, parietobasilares e basilares dos mesentérios. O esfíncter marginal, quando presente, está localizado logo abaixo ou um pouco abaixo da margem distal da coluna, sendo uma camada de musculatura circular concentrada, que pode ser revestida por endoderme ou mesogléia e cuja função primária é dobrar a margem da coluna por cima dos tentáculos durante a retração do pólipo. Sua presença, localização (endoderme ou mesogléia) e forma são caracteres taxonômicos. Os músculos retratores encontram-se nas faces internas dos pares de mesentérios (exceto nos mesentérios diretivos, no qual estão nas faces externas dos pares), e se ligam ao disco oral e à base da anêmona com a função principal de puxar a parte distal do animal para baixo quando necessário. O músculo parietobasilar está na junção entre a base do mesentério e a coluna do pólipo. Suas fibras são diagonais e, geralmente, formam uma aba em cada lado do mesentério que pode variar em seu comprimento e espessura. Por fim, a musculatura basilar está na extremidade proximal dos mesentérios, paralelamente à inserção deste no disco pedal. A direção das fibras desse músculo é horizontal e auxiliam na fixação do pólipo ao substrato. Espécies que se enterram (como em Edwardsiidae - ver Daly \& Ljubenkov, 2008) podem apresentar a musculatura parietal. Esta está na junção do mesentério com a coluna, mas se difere da musculatura parietobasilar por ser simétrica, não formar abas, e por seus feixes serem longitudinais. Outras descrições de morfologia de anêmonas do mar com características relevantes para a taxonomia podem ser encontradas em Stephenson (1928), Carlgren (1949), Corrêa (1964) e Shick (1991).

Dentro dos hexacorais, as anêmonas estão agrupadas na ordem Actiniaria Hertwig, 1882 (com 1.100 espécies válidas, segundo Fautin et al., 2007), para a qual diferentes autores apresentam distintas propostas classificatórias (e.g. Stephenson, 1921; Carlgren, 1949; 
Schmidt, 1974, Rodríguez et al., 2014). A proposta de classificação sugerida por Carlgren é historicamente a mais aceita (Daly et al., 2007), e divide as anêmonas em três subordens: Protantheae Carlgren, 1891, Endocoelantheae Carlgren, 1925 e Nynantheae Carlgren, 1899. Os caracteres morfológicos utilizados na separação das subordens são: grau de desenvolvimento do disco pedal e musculatura basilar; presença e tipo de esfíncter; presença ou ausência de tratos ciliados nos filamentos mesentéricos; e arranjo dos mesentérios. No entanto, Rodríguez et al. (2014) sugerem que esta classificação pode não refletir a história evolutiva do grupo e, baseado em dados moleculares, propuseram a separação das anêmonas em duas subordens, Anenthemonae Rodríguez \& Daly, 2014 e Enthemonae Rodríguez \& Daly, 2014, que se diferenciam pelo arranjo dos mesentérios.

A monofilia de Actiniaria foi testada recentemente (Rodríguez et al., 2014), apesar de alguns trabalhos já terem indicado que seus agrupamentos são polifiléticos, inclusive grande parte das famílias (e.g. Bernston et al., 1999; Won et al., 2001; Daly et al., 2003, 2008; Rodríguez et al., 2012). De acordo com a análise de Rodríguez et al. (2014), a ordem Actiniaria não é monofilética devido à espécie Relicanthus daphneae (Daly, 2006) (que antes pertencia à infraordem Boloceroidaria Carlgren, 1924, dentro de Nynantheae) ter sido recuperada como grupo irmão de um clado que contém os membros da ordem Zoanthidea. Neste cenário, Actiniaria é caracterizada pelas seguintes características, sendo nenhuma delas exclusivas para a ordem: ausência de esqueleto, pólipos solitários, presença de apenas um ciclo marginal de tentáculos (Rodríguez et al. 2014). Um possível caráter fenotípico para os membros da ordem, o que também depende do posicionamento de $R$. daphneae, é a presença de abas apicais nos nematocistos, que consistem em três elementos triangulares no ápice das cápsulas que se dobram para fora no momento do disparo (ver Reft \& Daly, 2012; Rodríguez et al., 2014). 
A subordem Enthemonae contém a maioria das espécies de Actiniaria, incluindo a maioria dos membros de Nynantheae (sensu Carlgren, 1949), e seus representantes apresentam o típico arranjo de mesentérios dos actiniários: ciclos de mesentérios dispostos de forma hexâmera, sendo que os pares novos de mesentérios surgem das exoceles (Rodríguez et al., 2014). Dentro desta subordem estão contidas três superfamílias: Actinostoloidea Carlgren, 1932, Metridioidea Carlgen, 1893 e Actinioidea Rafinesque, 1815. Esta última compreende espécies que apresentam musculatura basilar e esfíncter marginal endodérmico ou ausente. Além disso, a maioria dos membros habita ambientes rasos, e grande parte apresenta algum tipo de protuberância na coluna ou margem (vesículas, verrugas, esférulas marginais, etc.) (Rodríguez et al., 2014). Actinioidea inclui membros da subtribo Endomyaria Stephenson, 1921 (sensu Carlgren, 1949; inclui espécies com musculatura basilar - tribo Thenaria - e que possuem esfíncter marginal endodérmico) e algumas famílias da tribo Athenaria Carlgren, 1899 (sensu Carlgren, 1949; maioria das espécies de anêmonas sem musculatura basilar). Carlgren (1949) dividiu Endomyaria em famílias sem a intenção de refletir a filogenia do grupo, de forma que os caracteres morfológicos escolhidos geraram agrupamentos artificiais em estudos posteriores (Daly et al., 2008; Rodríguez et al., 2012, 2014), apesar de muitas das famílias ainda serem válidas (Fautin, 2013).

\section{A família Actiniidae}

A família Actiniidae Rafinesque, 1815 compreende 44 gêneros e mais de 200 espécies (Daly et al., 2007), sendo um dos maiores grupos da ordem. Constitui a família melhor representada em levantamentos faunísticos realizados no mundo, inclusive no Brasil (e.g. Zamponi et al., 1998; Amaral et al., 2000), e seus representantes são encontrados principalmente em ambientes de águas rasas e costões rochosos (Häussermann, 2004). Apesar 
disso, assim como no restante da ordem Actiniaria, a distinção de gêneros e espécies dentro de Actiniidae é bastante complicada e muitos grupos necessitam ser reexaminados (Daly, 2003, 2004; Daly et al., 2008; Gomes et al., 2012).

Os representantes da família são em geral pólipos relativamente grandes (diâmetro maior que $2 \mathrm{~cm}$ ) e algumas espécies apresentam características diagnósticas facilmente observadas. Projeções da coluna são umas das características mais relevantes para a sistemática do grupo. Tradicionalmente, estruturas na coluna como verrugas, vesículas, acrorrágios e pseudoacrorrágios são usados para distinguir os gêneros de Actiniidae, enquanto caracteres histológicos e distribuição dos tipos de nematocistos separam as espécies (Stephenson, 1921, 1935; Carlgren, 1949; Häussermann, 2004). No entanto, a simplicidade do pólipo faz com que exista um baixo número de características taxonomicamente informativas, e a escassez de descrições detalhadas e chaves de identificação dificulta o reconhecimento da grande maioria das espécies (Daly, 2003; Häussermann, 2004)

Estudos propondo filogenias moleculares para Actiniaria sugerem a parafilia da família Actiniidae (Won et al., 2001; Daly et al., 2003, 2008, Rodríguez et al., 2014), e uma das principais causas apontadas é o fato da família ser definida, assim como de toda a ordem, com base na ausência de características ao invés da utilização de sinapomorfias (Daly et al. 2008; Gusmão \& Daly, 2010). Outro problema encontrado nesta família é a interpretação das projeções das colunas que, além de apresentarem uma grande plasticidade (Daly, 2003, 2004; Gomes et al., 2012), são geralmente difíceis de diferenciar em espécimes preservados (Häussermann, 2004). Devido a isso, as definições dos diferentes tipos de projeções da coluna são controversas o que torna difícil a tarefa de inferir uma direção para a evolução desses caracteres (Daly, 2003, 2004). 


\section{Gênero Bunodosoma}

Segundo Fautin (2013), o gênero Bunodosoma Verrill, 1899 apresenta atualmente 13 espécies válidas. Estas são, em sua maioria, de águas tropicais e subtropicais e ocorrem em ambas as costas do oceano Atlântico, na costa leste do Oceano Pacífico e no Indo-Pacífico (Fautin, 2013; Gomes et al., 2012). Gomes et al. (2012) descreveram uma nova espécie para o gênero, Bunodosoma zamponii Gomes, Schama \& Solé-Cava, 2012, encontrada na costa da Argentina, e que até então era identificada equivocadamente como Phymactis papillosa (Lesson, 1830) (Gomes et al., 2012). Portanto, as espécies válidas do gênero Bunodosoma são: Bunodosoma biscayense (Fisher, 1874), Bunodosoma caissarum Corrêa in Belém, 1987, Bunodosoma californicum (Carlgren, 1951), Bunodosoma cangicum Corrêa in Belém \& Preslercravo, 1973, Bunodosoma capense (Lesson, 1830), Bunodosoma cavernatum (Bosc, 1802), Bunodosoma diadema (Drayton in Dana, 1846), Bunodosoma fallax (Pax, 1922), Bunodosoma goanense den Hartog \& Vennam, 1993, Bunodosoma grande (Verrill, 1869), Bunodosoma granuliferum (Le Sueur, 1817), Bunodosoma kuekenthali Pax, 1910, Bunodosoma sphaerulatum Duerden, 1902 e B. zamponii.

Os representantes do gênero Bunodosoma são caracterizados pela presença de um esfíncter marginal circunscrito e endodérmico, acrorrágios, vesículas não aderentes e mesentérios férteis com musculatura bem desenvolvida (Haussermann, 2004; Gomes et al., 2012). A presença de acrorrágios e vesículas não aderentes, as principais características de morfologia externa utilizadas na identificação deste gênero, são compartilhadas com os gêneros Phymactis Milne-Edwards, 1857 e Phymanthea Carlgren, 1959, e têm sido a principal causa de identificações equivocadas (Häussermann, 2004; Gomes et al., 2012). A relação filogenética entre os três gêneros ainda não foi explorada. 
As semelhanças entre os gêneros Anthopleura Duchassaing de Fobressin \& Michelotti, 1860 e Bunodosoma foram exploradas em trabalhos direcionados para o estudo do primeiro gênero (e.g. Daly, 2004; Heestand, 2009). Segundo Daly (2004), Anthopleura é atualmente reconhecido pela presença de acrorrágios e verrugas adesivas. Daly (2003) infere que o acrorrágio é homólogo entre os membros dos gêneros Actinia Linnaeus, 1767, Anemonia Risso, 1826, Anthopleura e Bunodosoma, e outros trabalhos sugerem que Anthopleura é parafilético com relação a Bunodosoma (McCommas, 1991; Daly, 2004). Segundo Daly (2004), as vesículas arredondadas e não adesivas são uma característica derivada compartilhada (sinapomorfia) de Bunodosoma, enquanto as verrugas adesivas, encontradas em Anthopleura, seriam a condição primitiva compartilhada (simplesiomorfia) deste caráter.

Häussermann (2004) fornece uma lista de questões dentro do gênero Bunodosoma que necessitam ser analisadas. Segundo a autora algumas das espécies atualmente válidas necessitam ser reexaminadas, pois podem pertencer a outro gênero, como é o caso de $B$. capense (Lesson, 1830). B. fallax (Pax, 1922) tem um status desconhecido, pois a autora afirma que o material depositado em coleção não permite identificar o espécime como sendo pertencente ao gênero Bunodosoma. Já a espécie B. biscayense pode pertencer ao gênero Anthopleura (Häussermann, 2004; Daly, 2004), pois apresenta atributos anatômicos característicos desse gênero: os acrorrágios e as verrugas adesivas. O problema da espécie $B$. cavernatum (Bosc, 1802) foi apontado por McCommas (1991), que observou espécimes capazes de aderir pedaços de conchas quebradas às suas "vesículas" que, quando analisadas em cortes finos, mostram a aparência esperada de uma verruga adesiva.

O gênero Bunodosoma é comumente representado nas propostas de filogenia que utilizam dados moleculares (McCommas, 1991; Daly, 2004; Daly et al., 2008; Rodríguez et al., 2014), no entanto, a amostragem taxonômica é bastante restrita. McCommas (1991) 
incluiu B. cavernatum, B. californicum e B. granuliferum em suas análises, enquanto a análise de Daly (2004) incluiu as duas primeiras espécies e B. biscayense. Daly et al. (2008) e Rodríguez et al. (2014) incluíram apenas B. grande (Verrill, 1869) em sua análise da ordem Actiniaria. As espécies brasileiras do gênero, B. cangicum e B. caissarum, e a argentina $B$. zamponii foram incluídas apenas nas análises de Gomes et al. (2012). Ou seja, os problemas do gênero não foram foco de nenhum trabalho e o conhecimento de sua diversidade é limitado por problemas taxonômicos e de identificação.

\section{Cnidocistos como caracteres taxonômicos}

A utilização das cnidas (espirocistos, pticocistos e nematocistos) como ferramenta para estudos filogenéticos em Cnidaria sempre entusiasmou os pesquisadores principalmente devido à complexidade e variação encontrada nestas organelas, o que contrasta com a simplicidade estrutural da maioria dos representantes do filo (Fautin, 2009). Os estudos para a diferenciação de nematocistos (onde a variabilidade é maior) em Cnidaria iniciaram com trabalhos em Actiniaria de Möbius (1866), Bedot (1896) e Will (1909 apud Shostak et al., 2005) e na ordem Trachymedusae com Iwanzoff (1895 apud Shostak et al., 2005). Contudo, Weill (1934) foi o primeiro a propor uma classificação formal baseando-se na forma e tamanho das cápsulas dos nematocistos e da ornamentação (espinhos) que pode estar presente ou não nos filamentos. O sistema de classificação proposto por Weill (1934) sofreu diversas modificações desde sua publicação (e.g. Werner, 1965; Mariscal, 1974; England, 1991; Östman, 2000). Especificamente para Anthozoa, Schmidt (1972, 1974) criou uma nova proposta ao realizar um estudo detalhado dos nematocistos levando em conta as variações dos arranjos dos espinhos na base do túbulo do nematocisto. Schmidt (1974) propôs uma filogenia para Anthozoa baseando-se em sua proposta de classificação dos nematocistos. Sua proposta 
de nomenclatura é até hoje utilizada por alguns autores e para alguns grupos específicos, como para as anêmonas Acontiaria (Acuña et al., 2003). No entanto, a terminologia proposta por Weill (1934), com suas subsequentes modificações, é a mais utilizada em trabalhos recentes de descrição de anêmonas (e.g. Rodriguez et al., 2009; Gusmão, 2010). Östman (2000) realizou a mais recente redefinição de categorias de nematocistos baseando-se principalmente na classificação de Weill e nas posteriores modificações realizadas por Carlgren (1940, 1945), Cutress (1955), Mariscal (1974) e Calder (1977 apud Östman, 2000). Estudos continuam sendo realizados para tentar melhorar a compreensão da evolução das formas dos cnidocistos (e.g. Reft et al., 2009).

Os representantes de Anthozoa apresentam menos tipos de nematocistos do que os medusozoários (Fautin, 2009), mas, apesar disso, as cnidas são parte essencial da sistemática deste primeiro táxon. Segundo a proposta de classificação de Weill (1934) os diferentes tipos de nematocistos podem ser divididos em 16 categorias agrupadas em dois conjuntos mais amplos: haplonemos e heteronemos. Os haplonemos se caracterizam por apresentarem filamento e espinhos (quando presentes) sem distinção de regiões, enquanto os heteronemos sempre possuem espinhos e um espessamento basal no filamento denominado shaft (ressaltase que na literatura em língua portuguesa raramente se utilizam termos específicos para as diferentes partes dos nematocistos, e, quando isso ocorre, preferencialmente se utilizam os termos em inglês). Dentre os haplonemos há dois tipos: os átricos, que não apresentam espinhos, e os holótricos, que apresentam espinhos geralmente ao longo de todo o comprimento do filamento. O holótricos por sua vez podem ser isorriza ou anisorriza, sendo que estes últimos apresentam uma gradual, porém óbvia mudança no diâmetro ou nos espinhos ao longo de seu comprimento.

Entre os heteronemos há duas características importantes que definem a nomenclatura do nematocisto: o comprimento do filamento em comparação com a cápsula e a forma da 
região que conecta o shaft ao restante do filamento. O nematocisto é denominado macrobásico quando o shaft é mais longo que a cápsula (e encontra-se dobrado dentro da cápsula não disparada), e microbásico quando o shaft é mais curto que a cápsula. Se o shaft for mais largo que o restante do filamento e na junção entre as duas partes formar-se um funil em forma de $\mathrm{V}$, bastante característico na cnida não disparada, ele é denominado pmastigóforo. Caso essa forma de funil esteja ausente, ele será denominado b-mastigóforo ou basítrico, sendo que este último apresentará espinhos na base do shaft e o primeiro não. Caso ocorra um nematocisto $p$-mastigóforo em que não exista filamento após o shaft este é denominado amastigóforo. A modificação mais relevante proposta por Östman (2000) para esses nematocistos foi a modificação do termo amastigóforo para $p$-amastigóforo. Dessa forma geram-se os seguintes nomes para os nematocistos: átricos, holótricos isorrizas e anisorrizas, macrobásico p-mastigóforo, macrobásico b-mastigóforo, macrobásico $p$ amastigóforo, microbásico $p$-mastigóforo, microbásico b-mastigóforo, microbásico $p$ amastigóforo e basítrico. Todos esses tipos de cnidas são possíveis de serem encontrados em anêmonas do mar, além dos espirocistos.

Denomina-se cnidoma o conjunto de cnidocistos presentes nas diversas estruturas corporais de um indivíduo ou espécie de cnidário. Na descrição de uma espécie de anêmona do mar informações detalhadas referentes ao cnidoma são essenciais, tanto com relação aos tipos de cnidocistos encontrados quanto seus tamanhos (Fautin, 1988; 2009) e especifica-se o conjunto de cnidocistos para cada região do pólipo (tentáculos, coluna, actinofaringe, filamentos, especializações). Um dos motivos para isso é que a ordem Actiniaria é a que possui o cnidoma mais variado entre os demais Anthozoa (Schmidt, 1974). Em decorrência desse fato as cnidas se tornaram características taxonômicas importantes dentre as anêmonas não somente para definir táxons menos inclusivos como também para identificar espécies em 
grupos complexos (ver exemplos em Manuel, 1981). No entanto, a tradição em se dar um grande peso taxonômico ao cnidoma gera bastante discussão.

O valor dos dados de distribuição qualitativa (tipos de nematocistos) nos tecidos para definir espécies ou táxons superiores é constantemente questionado, pois se sabe que existem fatores biológicos que podem afetar as categorias de cnidas que uma espécie ou indivíduo apresenta. Um exemplo disso é o nematocisto do tipo holótrico: algumas espécies são definidas pela presença deste nematocisto em seus tentáculos ou em estruturas especializadas. No entanto, Watson \& Mariscal (1983) observaram que em Diadumene lineata (= Haliplanella luciae) eles aparecem em resposta a condições externas (agressão intraespecífica), quando há formação dos catch-tentacles. Além disso, há diversos casos de presença de uma determinada cnida em somente parte de uma população (Fautin, 1988). Já o uso de informações referentes ao tamanho das cnidas é ainda mais problemático. A apresentação destes valores é uma prática bem estabelecida, mas muitos autores discutem a importância destes dados (Fautin, 1988). Uma das principais justificativas para se a utilização destes é que se presume que as cnidas tenham seu crescimento determinado e, portanto, só são reconhecidas depois de estarem prontas, podendo ter um tamanho mais ou menos definido para uma determinada espécie (Fautin, 1988). No entanto, enquanto Ardelean \& Fautin (2004) observaram que o tamanho do nematocisto em um mesmo tecido tem tamanhos semelhantes, Acuña et al., (2003) registram a alta variabilidade nos tamanhos dos nematocistos entre indivíduos da mesma espécie. Além disso, já se demonstrou que o tamanho da cnida pode variar com a idade ou tamanho dos pólipos (Francis, 2004; Acuña et al. 2003) e com a variação em parâmetros ecológicos ou geográficos, como profundidade ou latitude (Zamponi \& Acuña, 1994). Como o tamanho das cnidas pode ser afetado por características intrínsecas ao indivíduo e pelas condições do hábitat, as informações quantitativas (tamanho) das cnidas são as que causam maior desconfiança entre os pesquisadores. 
Tantos impedimentos levam à conclusão de que não se deve dar um peso taxonômico muito grande para o cnidoma. No entanto, devido à existência de tão poucas alternativas de caracteres taxonômicos em anêmonas, o potencial dos cnidocistos como indicador de informações filogenéticas ou filogeográficas ainda deve ser estudado e melhor compreendido. Uma abordagem ainda pouco explorada para se delimitar variações interespecíficas, interpopulacionais e interindividuais é a integração de dados de morfologia do pólipo e cnidoma com dados moleculares. Poucas famílias ou gêneros têm sido foco de estudos filogenéticos (e.g. Gusmão, 2010) e estudos levando em conta as variações populacionais são extremamente escassos (e.g. Gomes, 2002). Neste contexto, o gênero Bunodosoma pode ser uma fonte de informações bastante interessantes para elucidar diversas questões, já que as espécies B. caissarum e B. cangicum apresentam distribuição ampla ao longo da costa brasileira, o que possibilita a observação de variações populacionais ao longo de uma ampla escala geográfica.

\section{Dificuldades no estudo das anêmonas do mar e o uso de dados moleculares}

O fato de as anêmonas apresentarem um pólipo bastante simples, aliado à elevada riqueza em espécies, faz com que os taxonomistas do grupo tenham dificuldade para estabelecer caracteres taxonômicos suficientes para serem utilizados na separação dos táxons (Fautin, 1988). Como comentado anteriormente, as descrições atuais de espécies de anêmonas incluem caracteres taxonômicos baseados na morfologia e anatomia do pólipo, além de informações sobre os tipos e medidas (variação e média do tamanho) das cnidas presentes em cada região do pólipo. Há, no entanto, controvérsias a respeito da forma com que os caracteres têm sido utilizados tanto na separação de espécies quanto de grupos em níveis hierárquicos mais elevados (Daly et al., 2007). 
Certos caracteres de morfologia do pólipo aos quais tem se dado valor taxonômico, como musculatura do esfíncter marginal e projeções da coluna, estão sujeitos a reavaliações ou são difíceis de serem distinguidos, o que torna sua utilização bastante problemática (Daly et al., 2007). Alguns autores vêm sugerindo novas formas de estudar as cnidas, tanto com relação às características morfológicas dos diferentes tipos de nematocistos, com a utilização da técnica de microscopia eletrônica de varredura e transmissão (e.g. Reft et al., 2009), quanto com relação à análise das medidas, com a utilização de tratamentos estatísticos mais adequados como os Modelos Lineares Generalizados (em inglês GLM) (Nelder \& Wedderburn, 1972) (e.g. Acuña et al., 2004). Esses dois tipos de abordagens ainda são pouco utilizadas nos estudos dentro do grupo e, portanto, sua eficácia na resolução dos problemas do estudo do cnidoma ainda não é bem conhecida.

Poucas famílias ou gêneros têm sido foco de estudos filogenéticos (e.g. Daly, 2004; Gusmão, 2010), e há uma grande necessidade de revisões de espécies e gêneros, para que se possa definir quais os limites entre a variação inter e intraespecífica (Rodriguez et al., 2012). A alta plasticidade morfológica encontrada em espécies de anêmonas aliada à falta de um consenso para a filogenia baseada em caracteres morfológicos faz com que a utilização de dados moleculares seja uma ferramenta importante (Daly et al., 2010). Portanto, no panorama atual dos estudos em Actiniaria é fundamental a realização de estudos que integrem informações morfológicas e dados moleculares para que seja possível a determinação de uma espécie de anêmona.

Estudos moleculares tem se mostrado particularmente interessantes para resolver questões de delimitação de espécies de anêmonas, pois muito do que se considera como sendo variação morfológica entre indivíduos de uma mesma espécie pode representar de fato diferenças entre espécies (Knowlton, 2000; Gomes et al., 2012). Problemas de definição de espécies em grupos que apresentam poucos caracteres taxonômicos e grande plasticidade 
morfológica ocorrem em outros grupos de Cnidaria, como, por exemplo, Zoantharia (Sinniger et al., 2008), Ceriantharia (Stampar et al., 2012) e Scyphozoa (Dawson, 2005).

A classe Anthozoa apresenta uma das taxas de relógio molecular mais baixas dentre os metazoários para marcadores mitocondriais (Shearer et al., 2002). Entretanto, a utilização de marcadores nucleares vem se mostrando extremamente efetiva para a delimitação de espécies em Anthozoa (Torres-Pratts et al., 2011; Stampar et al., 2012). A estimativa de divergências acumuladas nas regiões espaçadoras (ITSs) dos genes ribossomais (rDNA nuclear) permite, muitas vezes, a detecção de diferenças entre espécimes coespecíficos e portanto, pode ser uma ferramenta útil para o estudo de populações ou espécies proximamente relacionadas de antozoários (referências em Daly et al., 2010). A variação do marcador ITS foi explorada em alguns grupos de anêmonas até o momento, apresentando uma grande diversidade intraespecífica e, até mesmo, intragenômica nas espécies Diadumene lineata (Verrill, 1869) (Ting \& Geller, 2000) e Condylactis gigantea (Weinland, 1860) (Stoletzki \& Schierwater, 2005). Porém, estes marcadores apresentaram pouca variação intraespecífica em espécies dos gêneros Aulactinia Verrill, 1864 (Acuña et al., 2007) e Calliactis Verrill, 1869 (Gusmão, 2010), sendo, portanto, necessário que outros marcadores moleculares sejam explorados para reforçar ou se contrapor aos resultados encontrados para o marcador ITS.

As espécies endêmicas da América do Sul, B. caissarum e B. cangicum, apresentam uma ampla distribuição ao longo da costa brasileira e um estudo integrando informações morfológicas e moleculares em suas populações pode auxiliar na compreensão dos limites entre a variação interespecífica, entre populações e entre indivíduos de uma mesma espécie. Gomes (2002) estudou características morfológicas e genéticas (isoenzimas e ITS) de populações de B. cangicum, B. caissarum e B. zamponii, e relacionou estes fatores a características biológicas, ecológicas e de distribuição destas espécies. No atual estudo, complementamos seus resultados com amostragem mais ampla das espécies brasileiras, 
buscamos novos marcadores moleculares de interesse para estudos em nível populacional e específico em anêmonas do mar, e relacionamos isso com a variação do tamanho dos cnidocistos entre populações, um conjunto de estudos essencial para compreensão das variações entre espécies próximas e a variação intraespecífica em anêmonas do mar.

\section{Estrutura da Tese}

No Capítulo 1, apresento a revisão taxonômica do gênero Bunodosoma, realizada por meio de observações morfológicas, anatômicas e de cnidoma, e proponho uma hipótese filogenética para parte do gênero Bunodosoma, utilizando dados moleculares. No Capítulo 2, testo a viabilidade da utilização do marcador ITS nos estudos de populações de Bunodosoma, além de testar outros dois marcadores moleculares nucleares com o intuito de representar as diferenças genéticas existentes entre espécies, populações e indivíduos de uma mesma população de B. caissarum, B. cangicum e B. zamponii. No Capítulo 3, dados de morfometria dos cnidocistos são usados para buscar diferenças entre as populações de B. caissarum e $B$. cangicum. Desta forma, analisamos a variação dentro do gênero Bunodosoma e propomos que seus resultados sejam utilizados como modelo a ser testado na delimitação de espécies e populações de outras anêmonas do mar.

\section{Referências Bibliográficas}

Acuña, F. H.; Excoffon, A. C.; Zamponi, M. O. \& Ricci, L. 2003. Importance of nematocysts in taxonomy of acontiarian sea anemones (Cnidaria, Actiniaria): a statistical comparative study. Zoologischer Anzeiger 242: 75 - 81. 
Acuña, F. H.; Ricci, L; Excoffon, A. C. \& Zamponi, M. O. 2004. A novel statistical analysis of cnidocysts in acontiarian sea anemones (Cnidaria, Actiniaria) using generalized linear models with gamma errors. Zoologischer Anzeiger 243: 47 - 52.

Acuña, F. H.; Excoffon, A. C.; McKinstry, S. R. \& Martínez, D. E. 2007. Characterization of Aulactinia (Actiniaria: Actiniidae) species from Mar del Plata (Argentina) using morphological and molecular data. Hydrobiologia 592: 249 - 256.

Amaral, F. D.; Hudson, M.M.; da Silveira, F. L.; Migotto, A.E.; Pinto, S.M. \& Longo, L. 2000. Cnidarians of Saint Peter and St. Paul Archipelago, Northeast Brazil. In: International Coral Reef Symposium, 9. Bali: International Coral Reef Society 1: 567 -572.

Ardelean, A. \& Fautin, D. G. 2004. Variability in nematocysts from a single individual of the sea anemone Actinodendron arboretum (Cnidaria: Anthozoa: Actiniaria). Hydrobiologia 530/531: $189-197$.

Bedot, M. 1896. Note sur les cellules urticantes. Revue Suisse Zoologie 3: 533-539.

Berntson, E. A.; France, S. C. \& Mullineaux, L. S. 1999. Phylogenetic relationships within the class Anthozoa (phylum Cnidaria) based on nuclear 18S rDNA sequences. Molecular Phylogenetics and Evolution 13: 417 - 433.

Carlgren, O. 1940. A contribution to the knowledge of the structure and distribution of the cnidae in the Anthozoa. Kungliga Fysiografiska Sällskapets Handlingar 51 (3): 1 - 62.

Carlgren, O. 1945. Further contributions to the knowledge of the cnidom in the Anthozoa especially in the Actiniaria. Kungliga Fysiografiska Sällskapets Handlingar 56 (9): 1 - 24.

Carlgren, O. 1949. A Survey of the Ptychodactiaria, Corallimorpharia and Actiniaria. Kungliga Svenska Vetenskapsakademiens Handlingar 1(1): 1 - 121.

Corrêa D.D. 1964. Corallimorpharia e Actiniaria do Atlântico Oeste Tropical. Universidade de São Paulo, Ph.D Thesis, São Paulo, Brazil, 139 p.

Cutress, C.E. 1955. An interpretation of the structure and distribution of Cnidae in Anthozoa. Systematic Zoology 4: 120-137. 
Daly, M. 2003. The anatomy, terminology, and homology of acrorhagi and pseudoacrorhagi in sea anemones. Zoologishe Mededelingen 345: 89 - 102.

Daly, M. 2004. Phylogeny and biogeography of Anthopleura in the North Atlantic Ocean. Hydrobiologia 530/531: $241-248$.

Daly, M.; Fautin, D. G. \& Cappola, V. A. 2003. Systematics of the Hexacorallia (Cnidaria: Anthozoa). Zoological Journal of the Linnean Society 139: 419 - 437.

Daly, M.; Brugler, M. R.; Cartwright, P.; Collins, A.G.; Dawson, M. N.; Fautin, D.; France, S. C.; McFadden, C.S.; Opresko, D. M.; Rodriguez, E.; Romano, S. \& Stake, J. 2007. The phylum Cnidaria: A review of phylogenetic patterns and diversity 300 years after Linnaeus. In: Zhang, Z.-Q. \& Shear, W.A. (Eds). Linnaeus Tercentenary: Progress in Invertebrate Taxonomy. Zootaxa 1668: 127 - 182.

Daly, M.; Chaudhuri, A.; Gusmão, L. \& Rodríguez, E. 2008. Phylogenetic relationships among sea anemones (Cnidaria: Anthozoa: Actiniaria). Molecular Phylogenetics and Evolution 48: 292 301.

Daly, M.; Gusmão, L. C.; Reft, A. B.; Rodríguez, E. 2010. Phylogenetic signal in mitochondrial and nuclear markers in sea anemones (Cnidaria, Actiniaria). Integrative and Comparative Biology 50 (3): $371-388$.

Daly, M. \& Ljubenkov, JC. 2008 Edwardsiid sea anemones of California (Cnidaria: Actiniaria: Edwardsiidae), with descriptions of eight new species. Zootaxa 1860: 1-27

Dawson, M. N. 2005. Renaissance taxonomy: integrative evolutionary analyses in the classification of Scyphozoa. Journal of the Marine Biological Association of the United Kingdom 85: 733 739.

England, K. W. 1991. Nematocysts of sea anemones (Actiniaria, Ceriantharia and Corallimorpharia: Cnidaria): nomenclature. Hydrobiologia 216/217: 691 - 697.

Fautin, D. G. 1988. Importance of nematocysts to actinian taxonomy. In: Hessinger, D. A. \& Lenhoff, H. M (Ed.). The Biology of Nematocysts. San Diego and other cities: Academic Press. p. 487 500. 
Fautin, D. G., Zelenchuk, T. \& Raveendran, D. 2007. Genera of orders Actiniaria and Corallimorpharia (Cnidaria, Anthozoa, Hexacorallia), and their type species. Zootaxa 1668: $183-244$.

Fautin, D.G. 2009. Structural diversity, systematics, and evolution of cnidae. Toxicon 54: 1054-1064. Fautin, D. G. 2013. Hexacorallians of the World. Avaliable at: <http://geoportal.kgs.ku.edu/hexacoral/anemone2/index.cfm> [Access on May, 10th , 2016]

Francis, L. 2004. Microscaling: Why larger anemones have longer cnidae. Biological Bulletin 207: $116-129$.

Gomes, P.B. 2002. Estudio de la vicariancia en algunas especies de anémonas de mar (Cnidaria, Actiniaria) del intermareal de Brasil y Argentina con el uso de datos morfológicos y genéticos. Ph.D Thesis - Facultad de Ciencias Exactas y Naturales, Universidad de Buenos Aires, Buenos Aires, Argentina. 101pp.

Gomes, P.B.; Schama, R. \& Solé-Cava. 2012. Molecular and morphological evidence that Phymactis papillosa from Argentina is, in fact, a new species of the genus Bunodosoma (Cnidaria: Actiniidae). Journal of the Marine Biological Association of the United Kingdom 92 (5): 895910.

Gusmão, L. C. 2010. Systematics and evolution of sea anemones (Cnidaria: Actiniaria: Hormathiidae) symbiotic with hermit crabs. Ph.D. Thesis - The Ohio State University, USA. 337 pp.

Gusmão, L. C. \& Daly, M. 2010. Evolution of sea anemones (Cnidaria: Actiniaria: Hormathiidae) symbiotic with hermit crabs. Molecular Phylogenetics and Evolution 56: 868 - 877.

Häussermann, V. 2004. Re-description of Phymactis papillosa (Lesson, 1830) and Phymanthea pluvia (Drayton in Dana, 1846) (Cnidaria:Anthozoa), two common actiniid sea anemones from the south Pacific with a discussion of related genera. Zoologische Mededelingen 78: 345-381.

Heestand, E.N. 2009. Phylogeny and Evolution of Anthopleura (Cnidaria:Anthozoa:Actiniaria). MSc dissertation - The Ohio State University. 42 pp.

Knowlton, N. 2000. Molecular genetic analyses of species boundaries in the sea. Hydrobiologia 420: 73-90. 
Manuel, R. L. 1981. British Anthozoa - Synopses of the British Fauna: new series 18. London: Academic Press. 241 pp.

Mariscal, R. N. 1974. Nematocysts. In: Muscatine, L. \& Lenhoff, H. M. (Eds.). Coelenterate Biology. Reviews and New Perspectives. New York: Academic Press. pp. 129 - 178.

McCommas, S.A. 1991. Relationships within the family Actiniidae (Cnidaria, Actiniaria) based on molecular characters. Hydrobiologia 216/217: 509-512.

Möbius, K.A., 1866. Ueber den Bau, den Mechanismus und die Entwicklung der Nesselkapseln einiger Polypen und Quallen. Abhandlungen aus dem Gebiete der Naturwissenschaften Ver. Hamburg V(1): 1-22, pls. 1-2.

Nelder, J. A. \& Wedderburn, R. W. M. 1972. Generalized Linear Models. Journal of the Royal Statistical Society 135 (3): 370- 384.

Östman, C. 2000. A guideline to nematocyst nomenclature and classification, and some notes on the systematic value of nematocysts. Scientia Marina 64 (Supl. 1): 31 - 46.

Reft, A. J.; Westfall, J. A. \& Fautin, D. G. 2009. Formation of the apical flaps in nematocysts of sea anemones (Cnidaria: Actiniaria). Biological Bulletin 217: 25 - 34.

Reft, A. \& Daly, M. 2012. Morphology, distribution, and evolution of apical structure of nematocysts in Hexacorallia. Journal of Morphology 273: 121-136.

Rodríguez, E.; López-González, P. J. \& Daly, M. 2009. New family of sea anemones (Actiniaria, Acontiaria) from deep polar seas. Polar Biology 32: 703 - 717.

Rodríguez, E.; Barbeitos, M.; Daly, M.; Gusmão, L.C.; Hausserman, V. 2012. Towards a natural classification: phylogeny of Acontiate sea anemones (Cnidaria, Anthozoa, Actiniaria). Cladistics 28: 375 - 392 .

Rodríguez, E; Barbeitos, MS; Brugler, MR; Crowley, LM; Grajales, A; Gusmão, L; Häussermann, V.; Reft, A. \& Daly, M. 2014. Hidden among Sea Anemones: The First Comprehensive Phylogenetic Reconstruction of the Order Actiniaria (Cnidaria, Anthozoa, Hexacorallia) Reveals a Novel Group of Hexacorals. PLoS ONE 9(5): e96998. doi:10.1371/journal.pone.0096998 
Shick J. M. 1991. A Functional Biology of Sea Anemones. London: Chapmann and Hall.

Schmidt, H. 1972. Die nesselkapseln der Anthozoen und ihre bedeutung für die phylogenetische systematik. Helgoländer wissenschaftliche Meeresuntersuchungen 23: 422 - 458.

Schmidt, H. 1974. On evolution in Anthozoa. Proceedings of the 2nd International Coral Reef Symposium 1: $533-560$.

Shearer, T.L.; van Oppen, M. J. H.; Romanos, S. L. \& Wörheide, G. 2002. Slow mitochondrial DNA sequence evolution in the Anthozoa (Cnidaria). Molecular Ecology 11: 2475-2487.

Shostak, S. 1993. A symbiogenetic theory for the origins of cnidocysts in Cnidaria. Biosystems 29: 4958.

Sinniger, F.; Reimer, J. D. \& Pawlowski, J. 2008. Potential of DNA sequences to identify Zoanthids (Cnidaria: Zoantharia). Zoological science 25: 1253 - 1260.

Stampar, S.N.; Maronna, M.M.; Vermeij, M.J.A.; Silveira, F.L. \& Morandini, A.C. 2012. Evolutionary diversification of banded tube-dwelling anemones (Cnidaria; Ceriantharia; Isarachnanthus) in the Atlantic Ocean. PLoS One 7: e41091.

Stampar, S.N., Maronna MM, Kitahara MV, Reimer JD \& Morandini AC. 2014. Fast-Evolving Mitochondrial DNA in Ceriantharia: A Reflection of Hexacorallia Paraphyly? PLoS ONE 9(1): e86612. doi:10.1371/journal.pone.0086612

Stephenson, T. A. 1921. On the classification of Actiniaria. Part II. Consideration of the whole group and its relationships, with special reference to forms not treated in Part I. Quarterly Journal of Microscopical Sciences 65: 493 - 576.

Stephenson, T.A. 1928. The British Sea Anemones - volume I. London: The Ray Society. 148 pp.

Stephenson, T.A. 1935. The British Sea Anemones - volume II. London: The Ray Society. 426 pp.

Stoletzki, N. \& Schierwater, B. 2005. Genetic and color morph differentiation in the Caribbean sea anemone Condylactis gigantea. Marine Biology 147: 747 -754.

Ting, J. H. \& Geller, J. B. 2000. Clonal diversity in introduced populations of an asian sea anemone in North America. Biological Invasions 2: 23 - 32. 
Torres-Pratts, H.; Lado-Insua,T.; Rhyne, A.; Rodriguez-Matos, L. \& Schizas, N. 2011. Two distinct, geographically overlapping lineages of the corallimorpharian Ricordea florida (Cnidaria: Hexacorallia: Rocordeidae). Coral Reefs 30: 391 - 396.

Watson, G. M. \& Mariscal, R. N. 1983. The development of a sea anemone tentacle specialized for aggression: morphogenesis and regression of the catch-tentacle of Haliplanella luciae (Cnidaria, Anthozoa). Biological Bulletin 164 (3): 506 - 517.

Weill, R. 1934. Contribution à l'etude des cnidaires et leurs nématocyctes I e II. Travaux de la Station Zoologique de Wimeraux 10 - 11: 1 - 701.

Werner, B. 1965. Die Nesselkapseln der Cnidaria, mit besonderer Beriicksichtigung der Hydroida. I. Klassifikation und Bedeulung für die Systematik und Evolution. Helgoländer Wissenschaftliche Meeresuntersuchungen 12: 1 - 39.

Won, J. H.; Rho, B. J. \& Song, J-I. 2001. A phylogenetic study of the Anthozoa (phylum Cnidaria) based on morphological and molecular characters. Coral Reefs 20: 39 - 50.

Zamponi, M. O. \& Acuña. F. H. 1994. La variabilidad de los cnidocistos y su importancia en la determinación de clines. Physis 49: 7 - 18.

Zamponi, M. O; Belém, M. J. C.; Schlenz, E \& Acuña, F. H. 1998. Distribution and some ecological aspects of Corallimorpharia and Actiniaria from shallow waters of the South American Atlantic coasts. Physis, Secc. A 55: 31 - 45. 


\section{Conclusão geral}

A partir das evidências disponíveis, pudemos concluir no Capítulo 1 que o gênero Bunodosoma engloba 14 espécies válidas e é parafilético em relação a outras espécies da Família Actiniidae. Além disso, os dados evidenciam que três espécies reconhecidas $(B$. biscayense, B. fallax e B. sphaerulatum) seguem apresentando problemas taxonômicos, e uma revisão mais detalhada focada nas mesmas é necessária. Em B. biscayense é necessário compreender melhor a natureza das projeções da coluna. Já para B. fallax e B. sphaerulatum é necessário que mais material seja coletado em suas localidades tipo para que possa se realizar estudos mais completos de seus aspectos de anatomia interna e cnidoma. Não há evidências até o momento que suportem o posicionamento de $B$. fallax como pertencente ou não a Bunodosoma. Bunodosoma sphaerulatum por outro lado apresenta caracteres que permitem sua alocação no gênero Bunodosoma, no entanto, existe a possibilidade de que os espécimes associados e está espécie sejam na realidade pólipos juvenis de alguma das outras espécies que ocorrem no mar do Caribe (B. cavernatum, B. granuliferum e B. kuekenthali).

Em nossa hipótese filogenética apresentada no Capítulo 1, as espécies $B$. cavernatum e B. granuliferum formaram um clado, porém, com nosso conjunto de evidências, não podemos afirmar nada acerca de seus relacionamentos internos, e o consenso estrito resulta em uma politomia. Associado a isso, ambas espécies são fenotipicamente muito similares, compartilhando características de morfologia externa, interna e, em grande parte, de cnidoma. Estas espécies são diferenciadas apenas por bandas/listras longitudinais presentes na segunda espécie e ausentes na primeira. Dessa forma, apenas com o uso de uma quantidade maior de evidências moleculares se pode testar de forma robusta a validade taxonômica dessas espécies. 
As espécies do Atlântico Sul, B. caissarum, B. cangicum e B. zamponii, também formaram um clado em nossa hipótese filogenética do Capítulo 1. Bunodosoma caissarum pode ser fenotipicamente e geneticamente (com o uso do marcador molecular ITS) diferenciada das outras espécies do clado. Já as espécies $B$. cangicum e B. zamponii são fenotipicamente semelhantes e mesmo com o uso do marcador molecular ITS (Capítulo 2) não podemos afirmar nada conclusivo acerca de suas relações, que devem ser mais bem exploradas em estudos futuros.

No capítulo 2 concluímos que os marcadores moleculares nucleares ITS (espaçador transcrito interno), ALG11(em inglês, asparagine-linked glycosylation 11 homolog ) e MAT (em inglês, methionine adenosyltransferase) não são apropriados para a realização de estudos populacionais no gênero Bunodosoma. Enquanto o primeiro marcador apresentou variação suficiente para separar B. caissarum de B. cangicum e B. zamponii, os outros dois marcadores não apresentaram variabilidade entre as espécies analisadas. Mais testes com outros marcadores nucleares ou técnicas alternativas, como as de sequenciamento de nova geração (NGS, sigla em inglês) necessitam ser realizados para que se possa encontrar um marcador que permita a realização de estudos filogeográficos em anêmonas do mar.

Por fim, no capítulo 3 conseguimos detectar alguns tipos de nematocistos que podem auxiliar no estudo de populações de B. caissarum e B. cangicum. Para a primeira espécie, o único tipo de cnidocisto que parece ter potencial para esses estudos são os basítricos dos tentáculos. Já para B. cangicum, os resultados dos seguintes nematocistos se destacaram: holótricos dos acrorrágios, basítricos dos tentáculos externos e microbásico b-mastigóforo dos filamentos. Sugerimos que os dados levantados para esses tipos detectados sejam analisados com maior detalhe, para que se possa compreender se realmente contribuem para estudos de população. Além disso, verificamos que na espécie B. cangicum, há diferenças significativas 
nos tamanhos dos basítricos e dos espirocistos dos tentáculos do ciclo mais externo e mais interno. Este resultado deve ser levando em conta em futuras descrições de anêmonas do mar.

De forma geral, podemos concluir que o conjunto de resultados do presente estudo contribui para um melhor conhecimento da sistemática e biologia das anêmonas do mar. É de grande importância que estudos integrativos sejam realizados com outros grupos desta ordem, para gerar subsídios para a compreensão de aspectos evolutivos da ordem Actiniaria. 